\title{
BEHAVIORAL MEASURES DURING VIRTUAL REALITY BASED MOTOR REHABILITATION
}

\author{
Beatriz Rey ${ }^{1}$, Alejandro Oliver ${ }^{1}$ and Jose M. Monzo ${ }^{2}$ \\ ${ }^{1}$ Departamento de Ingeniería Gráfica, Universitat Politècnica de València \\ Camino Vera, s/n, 46022, Valencia, Spain \\ ${ }_{2}^{2}$ Instituto de Instrumentación para Imagen Molecular (I3M), Centro Mixto CSIC - Universitat Politècnica de València \\ Camino Vera, s/n, 46022, Valencia, Spain
}

\begin{abstract}
Virtual reality is a technology that has been integrated in recent years in many motor rehabilitation protocols. It allows the patient to perform the training exercises in a virtual world controlling the movements of an avatar. Although exercises are usually adapted or modified by the therapist depending on the patients' evolution, VR systems do not usually include analytics to evaluate the performance of the exercises. In the present work, the goal is to design and develop a VR-based first-person perspective motor rehabilitation system that registers relevant performance measures during the training procedure. The developed application runs on a PC with an HTC Vive Pro virtual reality headset and Valve Index controllers. It has been developed using Unity 3D with OpenVR Unity integration. The exercise consists of moving an object from an initial to a final position in specific moments (iterations). The application registers information about the behavior and the performance of the participant during each iteration. The behavioral data that is monitored and saved during each training session will be analyzed and a new version of the application will be developed to provide the therapist with an analytics platform that can be considered to decide new steps of the training protocol.
\end{abstract}

\section{KEYWORDS}

Virtual Reality, Head Mounted Displays, Controllers, Tracking, Motor Training, Behavioral Measures

\section{INTRODUCTION}

Motor rehabilitation is a procedure that can be applied to different kinds of patients, such as people with motor disabilities, pain or stroke patients. After an initial evaluation, the therapist identifies the movements that are affected and decides the exercises that are important for the patients to recover or improve their motor function. The motor training usually takes place in a rehabilitation center, although, sometimes, it also includes exercises to be performed at home. The exercises can be adapted or modified depending on the patients' evolution during the rehabilitation procedure. To do that, it is very important to check if the exercises are being performed correctly.

Technologies such as virtual reality (VR) have been applied to increase the engagement or to complement at home. In fact, many VR devices have been made commercially available to the mass-market in recent years and have allowed a wider diffusion of this technology. Two technologies have been especially relevant for rehabilitation: head-mounted displays (HMDs) and motion capture or tracking systems (Perez-Marcos, 2018). Using these technologies, it is possible to create highly immersive experiences where it is possible to generate the illusion known as "embodiment" (Longo et al., 2008). VR hardware and software are used to substitute the person body by a virtual one, usually with the goal of generating subjective illusions of body ownership and agency (Kilteni et al., 2012) (Tsakiris, 2010) (Spanglang et al., 2014). Creating these illusions, especially in first-person perspective, is fundamental for the success of the rehabilitation (Gorisse et al., 2017).

The goal of the present work is to design and develop a VR-based first-person perspective motor rehabilitation system that registers relevant performance measures during the training procedure. This system will allow the therapist to evaluate the patients' performance based on the registered measures, so the protocol can be adapted depending on the evolution. 


\section{MATERIALS AND METHODS}

\subsection{Hardware}

The developed application runs on a PC composed of an Intel Core i7-9750H@2.60GHz and a Nvidia GeForce RTX 2060 graphics card. An HTC Vive Pro virtual reality headset is used to present the virtual environment to the patients. This system has a resolution of $2280 \times 1600$ pixels ( $1440 \times 1600$ pixels per eye), a field of view of $110^{\circ}$ and a refresh rate of $90 \mathrm{~Hz}$. Two base stations 2.0 are used, which allow to track up to $5 \mathrm{~m} \times 5 \mathrm{~m}$ roomscale space.

The controllers for interacting with the virtual reality application are the Valve Index Controllers. One of the main advantages of these devices is that they can be strapped to the participant hands during the exposure to the virtual environment (Figure 1). That way, participants do not need to hold them all the time, allowing them to perform more natural gestures. Their other advantage is that they can register the pressure that the user is applying during the gestures, which is a magnitude not measured by other commercially available controllers.

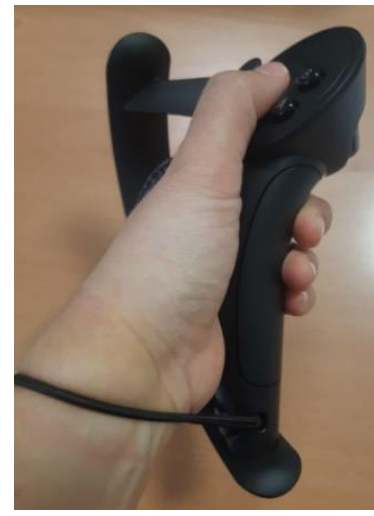

Figure 1. Left Valve Index Controller. The device is strapped to the user hand

Each controller includes different kinds of sensors to track hand position, individual finger position, motion and, as indicated, pressure. In the frontal part, several buttons are present: A and B buttons, the system button, a trackpad and a thumbstick. On the other part of the controller, the trigger button is placed. The main body of the controller is the grip force sensor, which is a sensitive region in the handle.

\subsection{Software}

The VR application has been developed using the real-time 3D engine Unity 3D, with the OpenVR Unity integration to connect and interact with the HTC Vive headset and with the Valve Index Controllers.

\subsection{VR Application}

The virtual environment is a room inside a house, with a window and several pieces of furniture distributed through the room. The participant visualizes the environment from a first-person perspective. The exercise consists of grabbing an object from its initial position and moving it to a new position. The objects appear in specific moments (iterations) detailed in an input Excel file that is read by the VR application. They should be grabbed with one of the hands and dragged to its new location (where they will disappear).

The therapist also visualizes the environment in the computer screen from the participant's point of view. The computer screen also shows a control panel. This control panel is only for the therapist, so it is not shown in the VR headset, just in the computer screen.

Before starting the session, the control panel allows the therapist to indicate the hand that is going to be used for the exercise: right, left or both. It also allows the therapist to select the input file that contains the temporal instants for the different iterations and the output file. The output file is an Excel file where 
information about the different iterations is saved, including data about the successfulness of each iteration and the behavioral data. Once the correct options are selected, the therapist will press a button and the training will start. During the training, the control panel will show information about the current iteration of the training and about the success in the previous iteration (Figure 2). The session finishes when the different iterations have been performed.

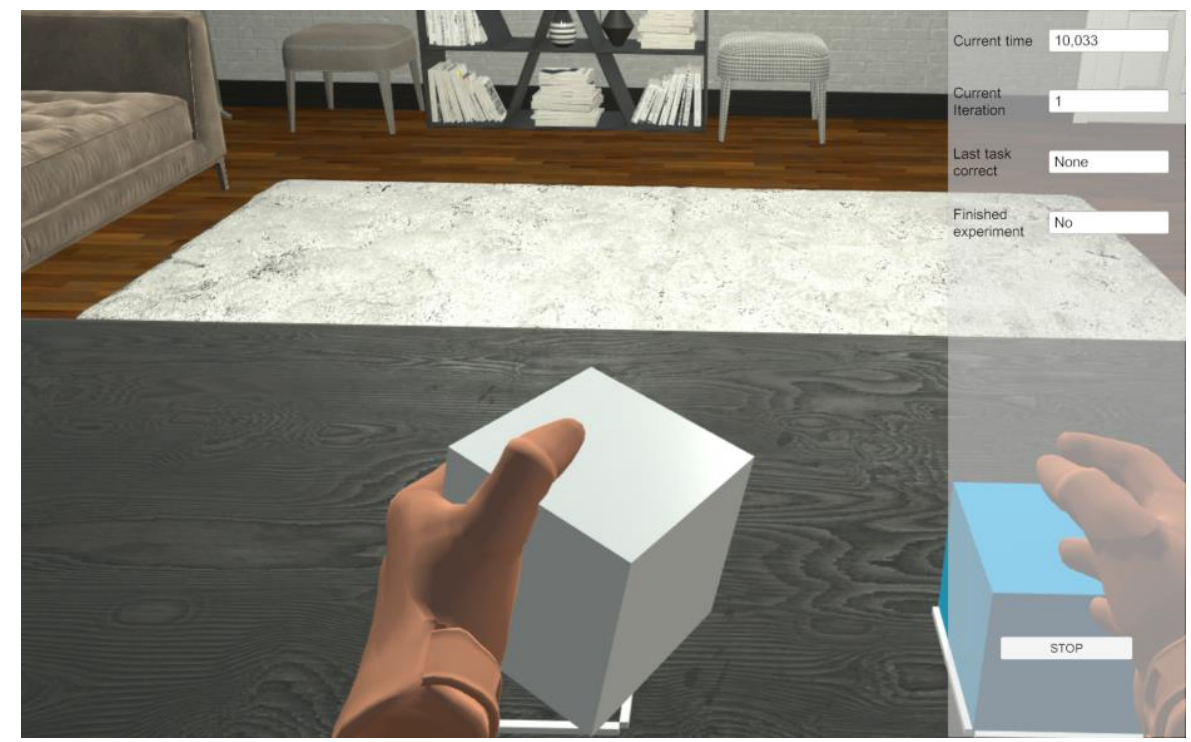

Figure 2. Therapist view of the environment during the training

\subsection{Behavioral Data}

The VR application registers information about the behavior and the performance of the participant during each iteration of the motor task. This information will be saved in different columns of the output Excel file.

Table 1. Information in the output Excel file. One row will be used for each iteration

\begin{tabular}{|c|c|c|}
\hline Column & Description & Units \\
\hline Iteration & Number of the iteration & \\
\hline Time & Time for the iteration & $\mathrm{s}$ \\
\hline Maximum hand height & $\begin{array}{l}\text { Maximum height of the hand during the trajectory to move } \\
\text { the cube }\end{array}$ & $\mathrm{cm}$ \\
\hline Grabbing number & $\begin{array}{l}\text { Number of times the object is grabbed by the participant } \\
\text { during the iteration }\end{array}$ & \\
\hline Object reaching time & $\begin{array}{l}\text { Elapsed time between the start of the iteration (when the } \\
\text { object appears in the scene) and the moment in which the } \\
\text { participant grabs the object }\end{array}$ & $\mathrm{s}$ \\
\hline Object holding time & Time during which the participant is holding the object & $\mathrm{s}$ \\
\hline Object scene time & Time during which the object is present in the scene & $\mathrm{s}$ \\
\hline Mean hand velocity & Mean hand velocity during the iteration & $\mathrm{m} / \mathrm{s}$ \\
\hline Maximum hand velocity & Maximum hand velocity during the iteration & $\mathrm{m} / \mathrm{s}$ \\
\hline Success & $\begin{array}{l}\text { It indicates if the iteration has been successfully finished } \\
\text { (Yes / No) }\end{array}$ & \\
\hline Grip Pressure & $\begin{array}{l}\text { Relative pressure in the grip area while holding the object } \\
\text { (0-minimum, 1-maximum) }\end{array}$ & \\
\hline Trigger pressure & $\begin{array}{l}\text { Relative pressure in the trigger button while holding the } \\
\text { object (0-minimum, 1-maximum) }\end{array}$ & \\
\hline Thumb & Indicates if the thumb is pressing (1) or not (0) & \\
\hline Index finger & Indicates if the index finger is pressing (1) or not (0) & \\
\hline Middle finger & Indicates if the middle finger is pressing (1) or not (0) & \\
\hline Ring finger & Indicates if the ring finger is pressing $(1)$ or not $(0)$ & \\
\hline Little finger & Indicates if the little finger is pressing (1) or not (0) & \\
\hline
\end{tabular}




\section{RESULTS AND CONCLUSION}

The prototype application has been developed and it is currently being tested with volunteers, using it to register behavioral parameters during a single VR-based motor training session. The behavioral data that is monitored and saved during each training session will be analyzed to acquire knowledge about the relations between each of the parameters and the success of the training.

The goal is that the current version of the system can be expanded and integrated in a more complete and complex protocol. In this new version, the relevant behavioral parameters will be registered and will be integrated in an analytics platform for the therapist where the evolution of the parameters can be graphically visualized and analyzed. This information will be used to decide when the patient is prepared to advance to the next stage of the protocol, and which exercises should be included in that stage. Although the current version of the system only includes exercises for upper limbs, full body tracking will also be integrated in the new version using the HTC Vive trackers, which are fully compatible with the system.

The use of a VR-based motor training protocol can be useful for different applications: motor rehabilitation, chronic pain patients, stroke patients, etc. Future studies will have to analyze possible differences in the relevant parameters depending on the kind of patients, and to evaluate their influence on the success of the protocol.

\section{ACKNOWLEDGEMENT}

This research was funded by Conselleria de Innovación, Universidades, Ciencia y Sociedad Digital, Comunitat Valenciana, Spain, grant number AICO/2019/029.

\section{REFERENCES}

Gorisse, G. et al, 2017. First- and third-person perspectives in immersive virtual environments: presence and performance analysis of embodied users. In Frontiers in Robotices and AI, Vol. 4.

Kilteni, K. et al, 2012. Extending body space in immersive virtual reality: a very long arm illusion. In PLOS ONE, Vol.7, No. 7, e40867.

Longo, M.R. et al, 2008. What is embodiment? A psychometric approach. In Cognition, Vol.107, No. 3, pp 978-998.

Perez-Marcos, D., 2018. Virtual reality experiences, embodiment, videogames and their dimensions in neurorehabilitation. In Journal of NeuroEngineering and Rehabilitation, Vol. 15, 113.

Spanglang, B. et al, 2014. How to build an embodiment lab: Achieving body representation illusions in virtual reality. In Frontiers in Robotics and AI, Vol. 1.

Tsakiris, M., 2010. My body in the brain: a neurocognitive model of body-ownership. In Neuropsychologia, Vol. 48, No. 3, pp. 703-712. 